\title{
Polarization and Belief Dynamics in the Black and White Communities: An Agent-Based Network Model from the Data
}

\author{
Patrick Grim, ${ }^{1,3}$ Stephen B. Thomas, ${ }^{2,6}$ Steven Fisher, ${ }^{3}$ Christopher Reade, ${ }^{4}$ Daniel J. Singer, ${ }^{5}$ Mary A. \\ Garza, $^{2,7}$ Craig S. Fryer ${ }^{2,7}$ and Jamie Chatman ${ }^{8}$ \\ ${ }^{1}$ Group for Logic \& Formal Semantics, Philosophy, Stony Brook University \\ ${ }^{2}$ Maryland Center for Health Equity, School of Public Health, Univ. of Maryland \\ ${ }^{3}$ Center for Study of Complex Systems, University of Michigan \\ ${ }^{4}$ Gerald R. Ford School of Public Policy, University of Michigan \\ ${ }^{5}$ Department of Philosophy, University of Michigan \\ ${ }^{6}$ Department of Health Services Administration, School of Public Health, University of Maryland \\ ${ }^{7}$ Department of Behavioral and Community Health, School of Public Health, University of Maryland \\ ${ }^{8}$ Department of Biostatistics, Graduate School of Public Health, University of Pittsburgh \\ pgrim@notes.cc.sunysb.edu
}

\begin{abstract}
Public health care interventions-regarding vaccination, obesity, and HIV, for example - standardly take the form of information dissemination across a community. But information networks can vary importantly between different ethnic communities, as can levels of trust in information from different sources. We use data from the Greater Pittsburgh Random Household Health Survey to construct models of information networks for White and Black communities-models which reflect the degree of information contact between individuals, with degrees of trust in information from various sources correlated with positions in that social network. With simple assumptions regarding belief change and social reinforcement, we use those modeled networks to build dynamic agent-based models of how information can be expected to flow and how beliefs can be expected to change across each community. With contrasting information from governmental and religious sources, the results show importantly different dynamic patterns of belief polarization within the two communities.
\end{abstract}

\section{Introduction}

Does information move differently in the Black community compared to the White community? What kinds of informational contacts link family and friends in the Black community? What are the levels of trust regarding information from personal contacts, from the government, and from church or religious leaders? What is the information network characteristic of the two communities, and what are the levels of trust in various information sources? Given different informational input to those networks, what can we expect the dynamics of belief formation and change to be in the two communities?

We use data from the Greater Pittsburgh Random Household Health Survey (Sellars, Garza, Fryer \& Thomas 2010) in order to construct models of information networks for White and Black communities, with levels of trust in various information sources correlated to position in those networks. With simple assumptions regarding belief change and social reinforcement, we use those social networks to build dynamic agent-based models of how information flows and beliefs change across each community. These modeling results, abstract in character and yet grounded in data, show that contrasting information from governmental and religious sources can be expected to produce importantly different configurations of belief and belief polarization within the two communities.

What we are after in the long term is an understanding of how public health interventions might utilize belief dynamics to optimize information flow across existing social networks. More specifically, the aim is to focus attention on the role of trust and distrust that drives the persistent problem of racial and ethnic disparities in health and health care (Smedley, Stith, \& Nelson 2003; Egede \& Zheng 2003; Chen, Fox, Cantrell, Stockdale, \& Kagawa-Singer 2007; Thomas \& Quinn 2008; Corbie-Smith, Thomas \& St. George 2002; Musa, Schulz, Harris, Silverman \& Thomas 2009; Rajakumar, Thomas, Musa, Almario, \& Garza 2009).

\section{Information Networks from the Data: Methods and Results}

The Greater Pittsburgh Random Household Health Survey was conducted for the University of Pittsburgh Research Center of Excellence on Minority Health and Health Disparities via telephone by International Communications Research (ICR), an independent research company. Interviews were conducted with 1018 respondents age 18 or older. Of those respondents, 671 self-identified as African American/Black and 347 as Caucasian/White.

The survey was a large one, with questions regarding selfesteem, social support, trust, experiences of discrimination, religious involvement, depression, violence, physical activity, and health issues. It was not originally designed for purposes of either network analysis or agent-based modeling, but there 
were several questions that allowed us to draw statistical data appropriate for these analyses. Among the questions asked of both Blacks and Whites, were two regarding social contact and support (Lubben, Gironda \& Lee 2001):

1. How many relatives do you feel at ease with that you can talk about private matters? Would you say:

None? One? Two? Three or four? Five through eight? Nine or more?

2. How many friends do you feel at ease with that you can talk about private matters? Would you say:

None? One? Two? Three or four? Five through eight? Nine or more?

We combined answers for the two questions, giving a selfestimated total for each individual of how many friends or family they felt at ease with talking about private matters. Do African Americans report a wider or narrower net of contacts than Whites? A different distribution of contact types? We developed an algorithm to give us arbitrary networks of 100 nodes which instantiated the degree distributions evident in the data. We have since found several other effective algorithms in the literature (Badham and Stocker 2010). An animation showing progressive approximation to a set of degree distributions from the data set can be seen at www.pgrim.org/belief_dynamics.

Figure 1 shows a histogram of degree distributions for the Black and White community. The top row of boxes represents the degree distributions drawn directly from the survey data. The bottom row of boxes shows the approximations to those distributions we are able to achieve in construction of our artificial networks. Figure 1 also shows the artificial networks themselves, with nodes ordered from center to periphery in terms of number of connections.

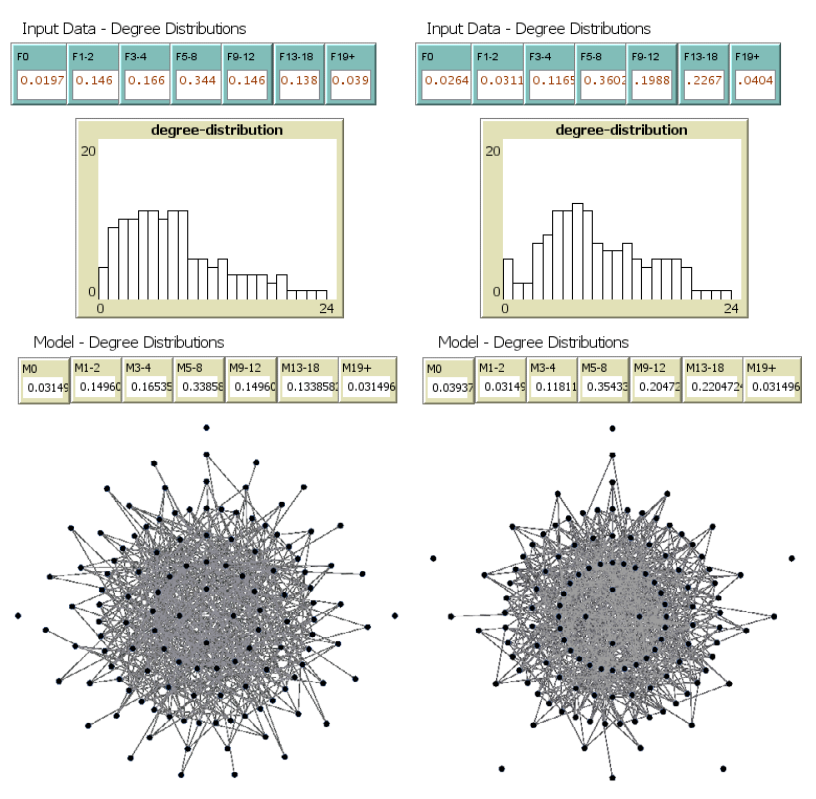

Figure 1 Friends and family networks in the Black (left) and White (right) communities.
Although a larger percentage of Whites report no family or friend contacts than Blacks do, a smaller percentage of Whites report only one or two friend or family contacts. The Black histogram offers a smoother curve, but shows a lower number of reported family and friend contacts over all. From the network diagrams it is evident that the Black information network is less tightly drawn: more nodes have fewer connections, and there are fewer numbers of nodes with large numbers of connections. Over all, the Black information network with family and friends appears to be sparser and more diffuse than that of the White community.

Although we have data on how many contacts each of our respondents reported, and although our model constructs a network that matches those numbers, our current data does not offer any information about other aspects of network structure - correlation coefficient, for example.

\section{Trust}

Our modeled networks reflect different patterns of information contact between friends and family within the contacts between friends and family within the White and Black communities. Information from those contacts can be expected to have a major impact on belief formation, but individuals also get information from other sources. The influence of information from any of these sources can be expected to vary with an individual's trust in the source.

One set of questions in the Greater Pittsburgh Random Household Health Survey targets trust. Among other sources, respondents were asked about their trust in information from the $\mathrm{CDC}$, friends or family, and church or religious leaders:

3. There are many people, or groups, from whom you might get information about health or health problems. For each of the following, please indicate how much you, personally, feel you would trust information that you got from that source.

How about the Center for Disease Control, sometimes referred to as the CDC? Would you say you:

Would trust definitely?

Would trust probably?

Would not trust probably?

Would not trust definitely?

Response options were the same for:

How about your friends or family?

How about your church or religious leaders?

For the sake of simplicity, we grouped 'would trust definitely' and 'would trust probably' as a positive trust category and 'would not trust probably' and 'would not trust definitely' as a negative trust category.

The initial presentation of data from the Greater Pittsburgh Random Household Health Survey gave trust levels across the full aggregate. Our agent-based model is more finely tuned than that. It's not as if there are two isolated facts:

(a) that some individuals in each community have a wider contact net of family and friends, and 
(b) that some individuals are more trusting of health information from particular sources.

There are correlations between these, evident in the raw data if not its initial presentation. We dug out those correlations in building the agent-based network model.

For those agents who reported no friend or family contacts, we incorporate appropriate percentages with positive or negative trust in information from various sources. For those reporting one or two contacts, we incorporate the different trust percentages appropriate to these, and so on.

Within the Black community, network diagrams of trust in information from (a) friends and family, (b) governmental sources, and (c) church or religious leaders are shown in Figure 2. Corresponding trust levels for each source of information for the White community are shown in Figure 3.
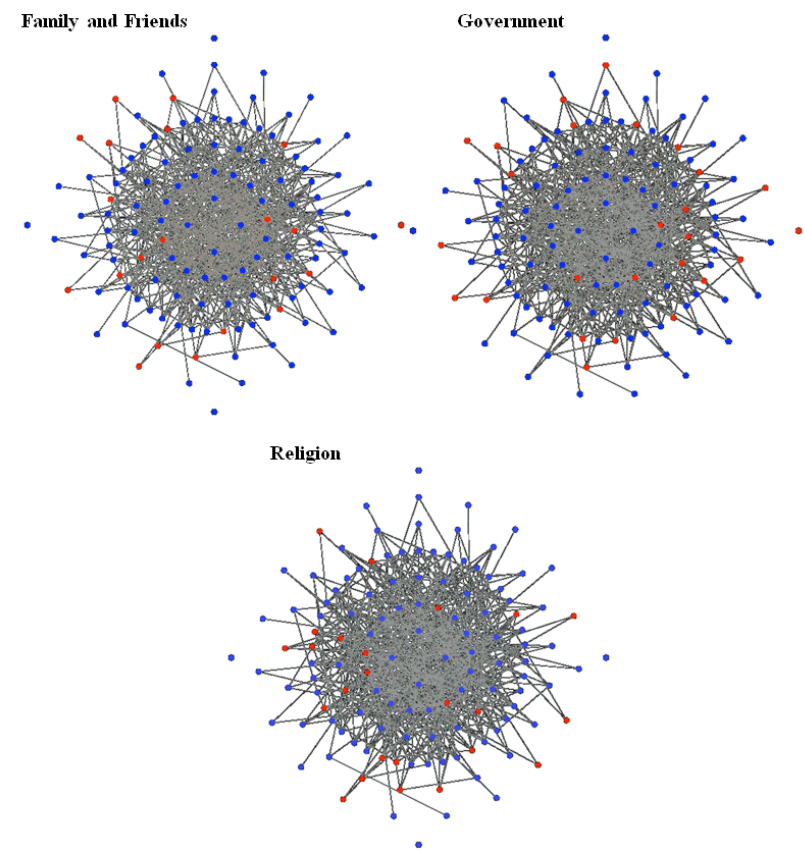

Fig. 2 Trust levels in information sources in the Black community. Blue $=$ positive trust. Red $=$ Distrust

Distrust of family and friends is tied more clearly to isolation from family and friends in the Black than in the White community. Many of those with only one or two contacts report distrust of family and friends within the Black community, whereas none of those with only one or two contacts do so in the White community. Distrust of government is more widespread within the Black community and is evident across most levels of connection.

Most noticeable, however, are differences in trust of church and religious leaders. Distrust of these information sources is much higher in the White community than in the Black community. Distrust of religious sources is also more strongly represented among those with many informational connections among the White compared to the Black community.

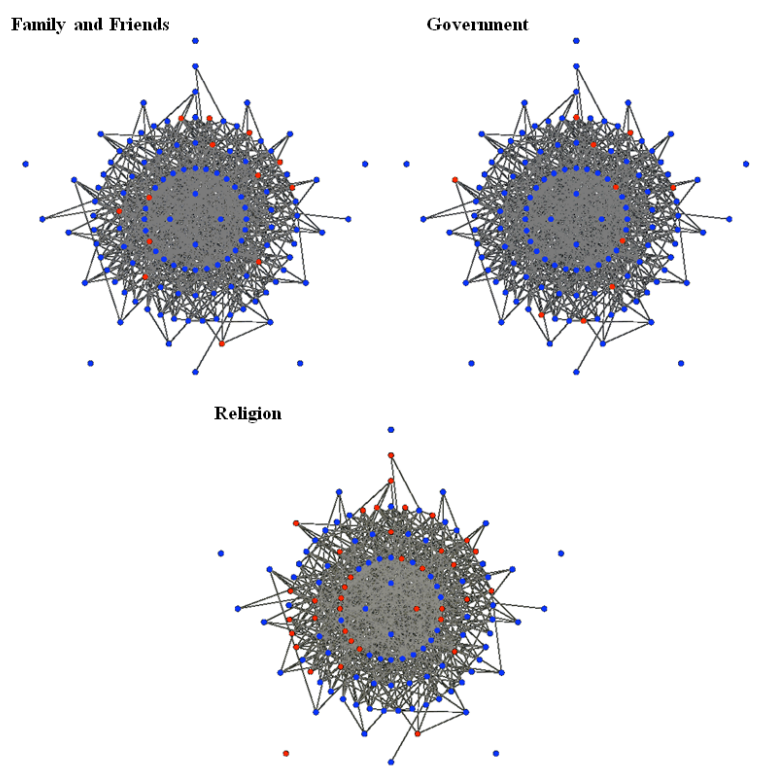

Fig. 3 Trust levels in information sources in the White community. Blue $=$ positive trust. Red $=$ Distrust

\section{Information Dynamics: Methods}

The networks constructed above, with correlated trust levels, allow us to project a dynamic model of belief across the two communities. Our aim is to offer an abstract model of how, given different information structures and different trust levels, the same information from external sources may result in different dynamics and different eventual configurations of community belief. How, for example, might conflicting health information from governmental and religious sources impact the dynamics and polarization of health care beliefs within the Black and White communities?

The data from which we have built the network model above is a snapshot of attitudes at a particular point in time. From that we can go on to construct a dynamic model, capable of offering a projection of potential changes in attitude over time. The fact that dynamic modeling can build on but also take us beyond static data carries pitfalls as well as promise. In order to test dynamic projections of the model in full we would need longitudinal data on changes in attitudes toward a particular health measure in the two communities correlated with data on information sources over the period at issue. That is longitudinal data we do not have and that we are unlikely to be able to get. In the absence of full longitudinal validation, we need to be particularly sensitive to the assumptions that drive dynamic projections.

A primary assumption in the construction of our dynamic model here is a mechanism for belief updating. We begin with the networks outlined above for each community: networks of contact which match degree distributions drawn from the data, correlated with trust levels regarding information from (a) friends and family, (b) governmental sources, and (c) church and religious leaders. What we want to know is how the structure of the information network and 
inputs from these sources affect the belief configuration of the community over time.

We model partial or gradational beliefs with numbers between 0 and 1 . These might represent the agents' degrees of confidence that they will catch a disease, for example, or their estimates of the severity of a disease (Harrison, Mullen, \& Green 1992; Janz \& Becker 1984; Mullen, Hersey, and Iverson, 1987; Strecher \& Rosenstock 1997). At the high end, these numbers might represent a belief that infection is imminent (represented by 1), which thereby warrants vaccination; at the low end, they might represent a belief that infection is impossible (represented by 0 ), and so vaccination is unwarranted; in such a case .5 might represent a neutral degree in between. Nothing in the model, however, indicates what types of belief are at issue or how the numerical scale is to be read. We abstract from the particular beliefs at issue, using numbers in their stead.

Agents update their beliefs, in our model, in light of information from family and friends, governmental sources, and church and religious leaders. How much they are influenced by each source will depend on how much trust they put in each source. At each step in the dynamic development of the model each agent considers input from (a) friends and family, weighted by how much trust he or she has in friends and family, input from (b) governmental sources, weighted by how much trust he or she has in government, and (c) from church and religious sources, again weighted by trust. These minimal assumptions, we can argue, are at least relatively realistic: people do have beliefs some of which can be represented on such a scale, and people are influenced to change those beliefs by, among other things, the expressed beliefs of those with whom they have contact and information that they trust from external sources. Given the networks of information contacts modeled above, it is clear that there will be reinforcement effects in such a dynamic. The fact that two trusting friends converge on a belief will strengthen that belief in both, for example. The fact that most of one's friends hold a belief will have a stronger effect than if only one does.

Our model starts, therefore, with a randomized distribution of beliefs. At each successive step, agents will have shifted their beliefs. They will then have different input from family and friends (though input from governmental and religious sources remain the same), producing a further modification of beliefs. What we track the change of belief and belief polarization over time in the two communities.

Although the general patterns of contact reinforcement and influence from outside forces can be seen as minimal and plausible assumptions of the model, the specific way in which these are instantiated in belief updating must be seen explicitly as modeling abstractions and simplifications. Our model is built on simplified assumptions regarding (1) the relative balance of various information sources and (2) the treatment of survey information on distrust. In this model, we use a simple weighted average in order to balance different information sources. Our basic updating algorithm is one in which current belief carries the largest weight in influencing later belief. Input from friends and family as a whole count half that weight in updating, with information from governmental and religious sources each counting one quarter. At each iteration, our agents average their current belief with input from each of these sources weighted in these proportions, resulting in their belief at the next iteration. That basic algorithm is altered slightly so as to indicate greater influence from greater numbers of contacts: for each of 5 categories of multiple friends (3-4, 5-8, 9-12, 13-18, and > 18) the influence of friends and family is increased by $10 \%$ over the base rate. The algorithm is also significantly altered by trust levels. In this model we simply discount sources an individual 'distrusts': governmental input to an individual who distrusts the government, for example, is simply ignored. In further studies we also explore interpreting reported distrust as a negative weighting for information from a particular source.

The updating algorithm we use, in the tradition of French 1956, Harary 1959, DeGroot 1974, and Golub \& Jackson 2010, 2011 and forthcoming, and is compatible with many standard accounts of partial belief dynamics including Bayesian conditionalization. In the most natural scheme for thinking of our agents' beliefs in Bayesian terms, there may be an expectation at the extremes, but see Hájek 2003. The use of weighted averaging in the updating algorithm could also been seen as a natural extension of the popular Equal Weight View in the literature on peer disagreement (Feldman 2006, Elga 2007, and Christenson 2007).

No-one thinks that weighted averaging of beliefs in an informational neighborhood-let alone these specific weights - captures the full psychological or normative dynamics of belief. Such a mechanism is a modeling abstraction intended to capture patterns of reinforcement which in some form clearly are plausible aspects of belief change. The more trusted an information source, the more likely information from that source is to change one's beliefs. The more one's beliefs are like those of one's network neighbors, and the more they are like more of one's network neighbors, the less inclination there will be to change those beliefs. The more one's beliefs are out of sync with one's neighbors, the greater the pressure there will be to change one's beliefs. That beliefs will change in accord with outside information and some pattern of reinforcement along those lines is very plausible, backed by a range of social psychological data, and is therefore an aspect of realism in the model. What is purely an assumption of the model is the particular algorithm used for reinforcement and informational influence - the particularly simple pattern of weighted belief averaging, applied homogeneously across agents.

In order to be informative regarding an exterior reality, a model, like any theory, must capture relevant aspects of that reality. In order to offer both tractability and understanding, a model, like any theory, must simplify. Our attempt is to capture some predictable but general aspects of belief change and reinforcement across a community; the admittedly artificial assumption of the specific algorithm we've used for belief updating is our simplification.

\section{Information Dynamics and Polarization in the Black and White Communities}

What can be projected for the Black community with belief change on this model and networks structure and trust levels derived from our data? How do beliefs change over time with particular governmental and religious inputs? 
Figure 4 shows the modeled development of beliefs across the Black community in terms of a histogram of the number of agents holding a belief in a particular category over time. In this case we use an input of ' 1 ' from governmental sources, ' 0 ' from religious sources, reflecting development in a case in which health care information from church and religious sources was directly opposed to that from the government. A full animation of such a development, correlated with node changes in the network, is available at www.pgrim.org/belief_dynamics.

The resultant belief configuration in this simulation has a mean of .48-slightly less than the mean of the random beliefs with which we began. It is the distribution of those beliefs that is particularly interesting, however. The result shows a clear central consensus, but development of the model shows increasing polarity, resulting in an obvious polarization at the two ends. If governmental sources say one thing and religious sources say another, our model indicates that the Black community will have a central consensus but a significant number of people with beliefs polarized at the extreme ends.

How does this development compare with the same inputs for the White community? Histograms of belief distribution over time for the White community are shown in Figure 5.

In this case the final mean for the community is .62 as opposed to .48. The model projection in a case of polarized information from governmental and religious sources is that governmental information will trump religious sources in the White community: belief in the White community will tend significantly toward that promulgated by the government. Within the Black community, in contrast, the two influences will be roughly on a par.

Here again, however, it is the distribution of beliefs that is equally or more important. In the White community the central consensus is significantly less sharp. In almost all runs it carries a secondary bump to the right of a central consensus, as shown here. Polarization at both extremes is significantly less in the White community: it is only the governmental end that shows a pile-up comparable to both ends in the Black community.

Our model therefore projects important differences in dynamics and final configuration of beliefs within the Black and White communities given the same polarized input from religious and governmental sources. Central consensus is more unified in the Black community, though with a more significant percentage of the population fully polarized and roughly equally balanced at the religious or governmental ends. The White community shows a less centralized consensus. Both in central areas and in polarized ends, it is governmental information that has a greater effect within the White community.

The results above use an input of '1' for governmental and ' 0 ' for religious informational sources. If both religious and governmental inputs are '1', progressive weighted averaging of inputs will drive consensus entirely to the ' 1 ' side. If both inputs are ' 0 ', that mechanism will drive consensus to the ' 0 ' side. The interesting results are therefore those in which we have differences in the two inputs. Our model can be run for any values of these, however, and need not be 'all or nothing'. With an input of .33 from one side and .66 on the other, a
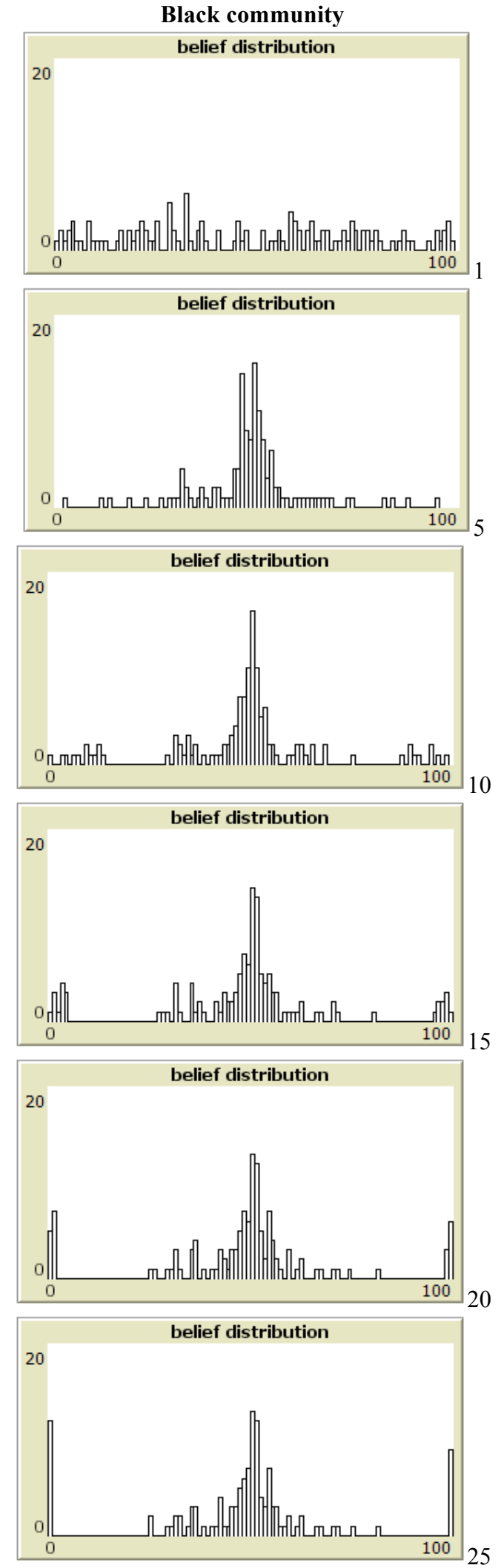

Fig. 4 Black community: dynamics of belief distribution given governmental input $=1$, religious input $=0$, iterations as numbered. 
Polarization and Belief Dynamics in the Black and White Communities: An Agent-Based Network Model from the Data
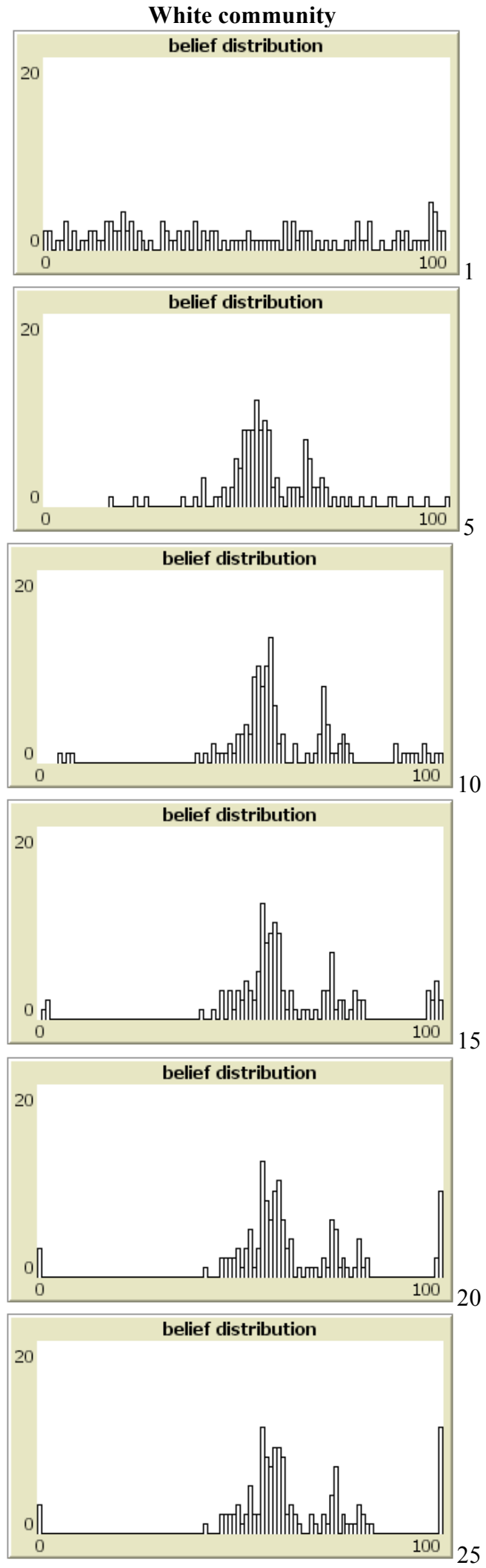

Fig. 5 White community: dynamics of belief distribution given governmental input $=1$, religious input $=0$, iterations as numbered. similar pattern of polarization is evident, but with the poles at .33 and .66 rather than at 0 and 1.

In Figure 6 we take comparison of runs for different inputs one step further. For each combination of inputs from religious and governmental sources, at 1 intervals, we ran 100 simulations and took medians and quartiles across those runs. The top landscape in Figure 6 shows the pattern of medians for the Black community across different inputs from governmental and religious sources. The lower landscape shows the corresponding pattern for the White community with that range of inputs. Together the two show the important tilt of the White community toward input from governmental sources when compared with the Black community.

\section{Black Community - Mean Belief with Religious and Governmental Inputs}

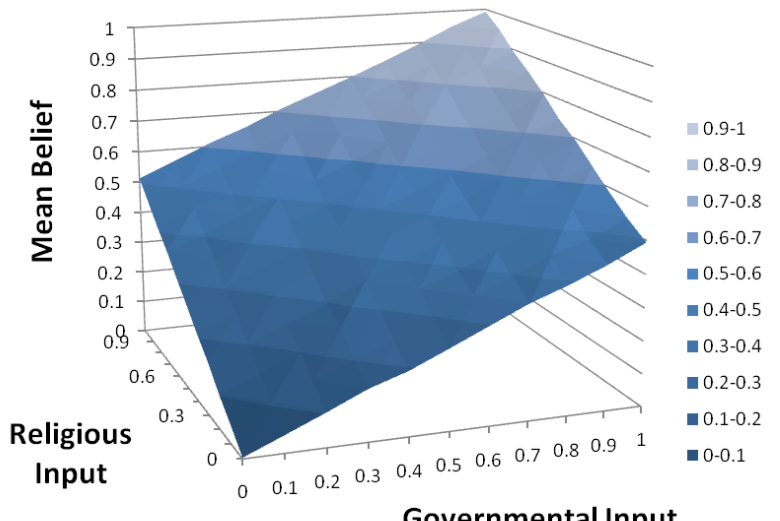

Governmental Input
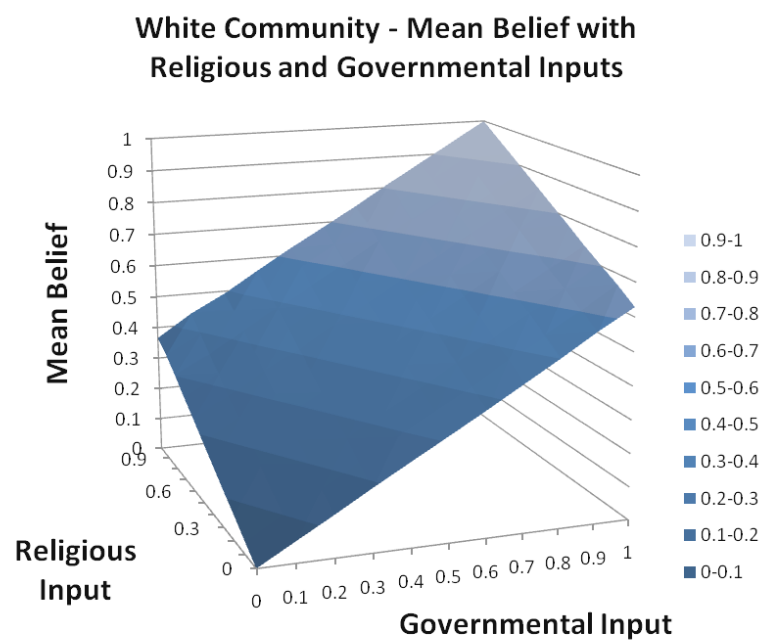

Fig. 6 Differences in median belief between the two communities across a range of different inputs from governmental and religious information sources.

A slice through the $(1,1)$ diagonal on each of these is shown in Figure 7, here including the 25th and 75th quartile for each 
population. This again makes vivid the differences in polarization between the communities at the same points of input from religious and governmental sources.
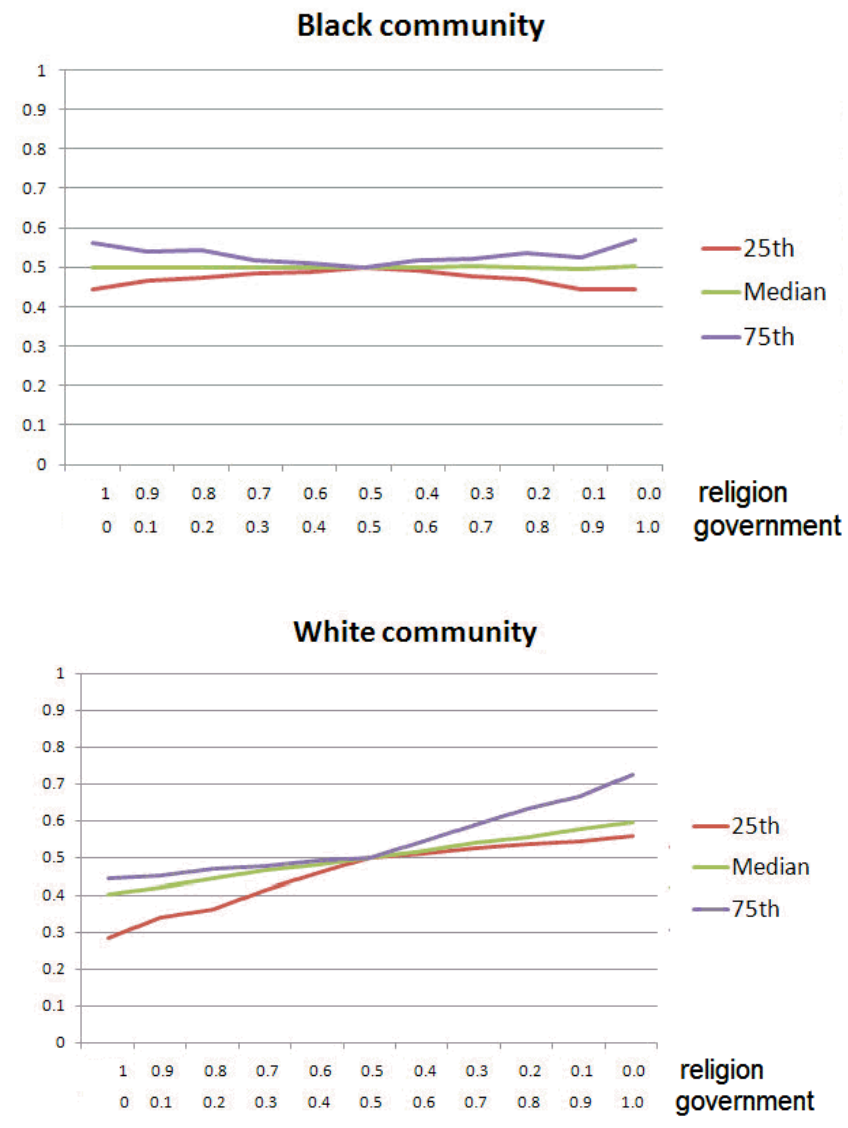

Fig. 7 Medians and quartiles for White and black

Communities with different combinations of input from governmental and religious information sources.

\section{Conclusion}

Dynamic agent-based modeling, constructed on social networks of interaction drawn from the actual data, demonstrates important divergences in social reaction to particular patterns of information within the Black and White communities. Surprise has often been expressed that Black and White communities have reacted differently given the same exterior information, particularly from governmental and or religious sources. The portrait of different social information structures offered here, incorporating network contact patterns that can differently amplify differences in trust, should reduce that element of surprise. This form of analysis can both offer a projection of differences in belief dynamics in future cases and might be used to best target effective information interventions in public health.

Our target is an understanding of the social dynamics of belief, a target we think clearly belongs under the wide umbrella of social epistemology. Because we want to understand the real social factors in belief formation, we've based our study in real data. In order to project longitudinal patterns from a static data snapshot, however, and in order to explore 'what ifs' relevant to normative questions of intervention, we've employed a range of simplifying assumptions within the techniques of dynamic agent-based modeling.

Passage of the historic Health Care and Education Reconciliation Act of 2010 (H.R. 4872) (US Congress 2010) and launch of Healthy People 2020 (US Dept. of Health \& Human Services 2009) provides an opportunity for multiple disciplines to collaborate on solutions to eliminate racial and ethnic health disparities. We believe this hybrid of disciplines and techniques can serve as an example for further research: work both data-driven and model-instantiated, both descriptive and normative, putting abstract techniques to the practical mission of eliminating health disparities and achieving health equity for all.

\section{Acknowledgements}

This work was supported in part by the National Institute of General Medical Sciences MIDAS (1U54GM088491-01) Computational Models of Infectious Disease Threats, and by a pilot grant for Developing an Agent-Based model to Assess Racial Differences in Medical Discrimination, Social Support and Trust, administered by the Graduate School for Public Health at the University of Pittsburgh. Work was also supported in part by the National Institute on Minority Health and Health Disparities (PG60MD000207, S. B. Thomas, PI) and the National Cancer Institute (K01CA148789, C. S. Fryer, PI and K01CA140358, M. A. Garza, PI). Grim is grateful for time at the Center for Complex Systems and the Department of Philosophy at the University of Michigan. Dr. Chatman's participation was supported in part by the Kellogg Health Disparity Scholars Program.

\section{References}

Badham, J., and Stocker, R. (2010). A spatial approach to network generation for three properties: Degree distribution, clustering coefficient and degree assortativity. journal of Artificial Societies and Social Simulation 13, http://jasss.soc.ac.uk/13/1/11.html>.

Chen, J., Fox, S., Cantrell, C., Stockdale, S., and Kagawa-Singer, M. (2007). Health Disparities and prevention: racial/ethnic barriers to flu vaccinations. Journal of Community Health 32, 5-20.

Christensen, D. (2007). Epistemology of disagreement: the good news. Philosophical Review 119: 187-217.

Corbie-Smith, G., Thomas, S. B., St. George, S. M. M. (2002). Distrust, race, and research. Archives of Internal Medicine 162: 2458-2463.

DeGroot, M. H. (1974). Reaching a consensus. Journal of the American Statistical Association 69, 118-121.

Egede, L. and Zhen, D. (2003). Racial/ethnic differences in influenza vaccination coverage in high-risk adults. American Journal of Public Health 93, 2074-2078.

Elga, A. (2007). Reflection and disagreement. Noûs 41: 478-502.

Feldman, R. (2006). Epistemological puzzles about disagreement. In Hetherington, S., editor, Epistemology Futures. Oxford: Oxford University Press, pp. 216-236.

French, J. (1956). A formal theory of social power. Psychological Review 63, 181-194.

Golub, B., and Jackson, M..O. (2010). Naive learning in social networks: Convergence, Influence, and the Wisdom of Crowds. American Economic Journal: Microeconomics 2, 112-149. 
Polarization and Belief Dynamics in the Black and White Communities: An Agent-Based Network Model from the Data

Golub, B., and Jackson, M. O. (forthcoming a). Network structure and the speed of leaning: Measuring homophily based on its consequences. Annals of Economics and Statistics. Available at http://papers.ssrn.com/abstract=1784542.

Golub, B., and Jackson, M. O. (forthcoming b). How homophily affects the speed of learning and best response dynamics. Quarterly Journal of Economics.

Harrison, J. A., Mullen, P., D., and Green, L. W. (1992). A meta-analysis of studies of the health belief model. Health Education Research 7: 107-116.

Hájek, A. (2003). What conditional probability could not be. Synthese 137 (3): 273-323.

Harary, F. (1959). A criterion for unanimity in French's theory of social power. In Cartwright, D.., editor, Studies in Social Power. Ann Arbor: University of Michigan Press.

Lubben, J. E., Gironda, M., and Lee, A. (2001). Refinements to the Lubben Social Network Scale: The LSNS-R. Behavioral Measurement Letter 7: 2-11.

Mullen, P. D., Hersey, J., and Iverson, D. C. (1987). Health behavior models compared. Social Science and Medicine 24: 973-981.

Musa, D., Schulz, R., Harris, R., Silverman, M., and Thomas, S. B. (2009). Trust in the health care system and the use of preventive health services by older black and white adults. American Journal of Public Health 99 (7): 1293-1299.

Rajakumar, K., Thomas, S. B., Musa, D., Almario, D., and Garza, M. (2009). Racial Differences in parents' distrust of medicine and research. Archives of Pediatric and Adolescent Medicine 163(2) 108-114.

Sellars, B., Garza, M. A., Fryer, C. S. and Thomas, S. B. (2010). Utilization of health care services and willingness to participate in future medical research: The role of race and social support. Journal of the National Medical Association 102: 776-786.

Smedley, B., Stith, A., and Nelson, A. (2003). Eds, Unequal Treatment: Confrontingf Racial and Ethnic Disparities in Health Care, Committee on Understanding and Eliminating Racial and Ethnic Disparities in Health Care, Institute of Medeicine of the National Academies, Academies Press.

Strecher, V., J. and Rosenstock, I. M. (1997). The health belief model. In Glanz, K., Lewis, F. M., and Rimer, B. K., Health Behavior and Health Education: Theory, Research and Practice, San Francisco: Jossey-Bass.

Thomas, S., and Quinn, S. (2008). Poverty and elimination of urban health disparities: challenge and opportunity. Annals of the New York Academy of Sciences 1136: 111-125. 